DRAFT VERSION NOVEMBER 18, 2018

Preprint typeset using LATEX style emulateapj v. 11/12/01

\title{
SWIFT OBSERVATIONS OF GRB 050128: THE EARLY X-RAY AFTERGLOW
}

\author{
S. Campana ${ }^{1}$, L.A. Antonelli ${ }^{2}$, G. Chincarini ${ }^{1,3}$, S. Covino ${ }^{1}$, G. Cusumano ${ }^{4}$, D. Malesani ${ }^{5}$, V. \\ Mangano $^{4}$, A. Moretti ${ }^{1}$, C. PAgani ${ }^{1,6}$, P. Romano $^{1}$, G. TAgliaferri ${ }^{1}$, M. CAPAlbi $^{7}$, M. Perri $^{7}$, P. $^{2}$

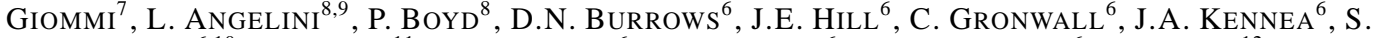

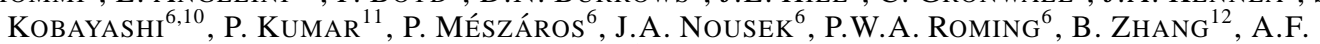 \\ ABBEY ${ }^{13}$, A.P. BEARdMORE ${ }^{13}$, A. BREEVELD ${ }^{14}$, M.R. GOAD ${ }^{13}$, O. GODET ${ }^{13}$, K.O. MASON ${ }^{14}$, J.P. \\ OSBORNE ${ }^{13}$, K.L. PAGE ${ }^{13}$, T. POOLE ${ }^{13} \&$ N. GEHRELS ${ }^{8}$ \\ campana@merate.mi.astro.it \\ Draft version November 18, 2018
}

\begin{abstract}
Swift discovered GRB050128 with the Burst Alert Telescope and promptly pointed its narrow field instruments to monitor the afterglow. X-ray observations started $108 \mathrm{~s}$ after the trigger time. The early decay of the afterglow is relatively flat with a temporal decay modeled with a power law with index $\sim-0.3$. A steepening occurs at later times $(\sim 1500 \mathrm{~s})$ with a power law index of $\sim-1.3$. During this transition, the observed X-ray spectrum does not change. We interpret this behaviour as either an early jet break or evidence for a transition from the fast cooling regime to the slow cooling regime in a wind environment.
\end{abstract}

Subject headings: gamma rays: bursts; X-rays: individual (GRB050128)

\section{INTRODUCTION}

The Swift Gamma-ray Burst Explorer (Gehrels et al. 2004) was successfully launched on 2004 November 20. Thanks to its fast-pointing capabilities, Swift is performing the first comprehensive observations of the early afterglow phase of GammaRay Bursts (GRB). A few GRBs have been followed by Swift within $200 \mathrm{~s}$ from their trigger time: GRB050117a (193 s), GRB050126 (131 s), GRB050128 (108 s), GRB050215b (108 s), GRB050219a (92 s) and GRB050315 (83 s).

In this paper we focus on GRB050128. The Burst Alert Telescope (BAT; Barthelmy et al. 2005) on board Swift triggered and located GRB050128 at 04:19:54 UT (Cummings et al. 2005). The burst profile is multi-peaked with a $T_{90} \mathrm{du}-$ ration of $13.8 \mathrm{~s}$. The fluence is $4.5 \times 10^{-6} \mathrm{erg} \mathrm{cm}^{-2}(15-$ $350 \mathrm{keV}$ ) making it a 'normal' burst with respect to the BATSE GRB population. The spectrum of the burst during the $T_{90}$ interval can be described by a power law model with photon index $\Gamma=1.5 \pm 0.1(15-350 \mathrm{keV})$. The peak energy is above $350 \mathrm{keV}$ making it a classical GRB.

Swift pointed autonomously to the GRB. We will report in the next sections about the XRT and UVOT observations. Ground-based follow-up observations started as soon as the GCN circular announcing the discovery of the new GRB was issued. This happened with some delay, since Swift was in the early phases of the mission and each circular was being checked manually before being distributed. The first GCN circular on GRB050128 was issued by the XRT team (Antonelli et al. 2005). The robotic 60-cm REM telescope located in La Silla pointed to GRB050128 approximately $3 \mathrm{hr}$ after the burst with good seeing conditions $\left(\sim 1^{\prime \prime}\right)$. No new sources were discovered with an upper limit of $H>17$ (Covino et al. 2005), $V>18.2, R>18.2$ and $I>17.9$ (Melandri et al. 2005). A further upper limit came from the 2-m Faulkes telescope South with $R>20.511 .5 \mathrm{hr}$ after the burst (Monfardini et al. 2005). GRB050128 has also been observed in the radio band at 8.4 $\mathrm{GHz}$ yielding an upper limit of $100 \mu \mathrm{Jy} \sim 11 \mathrm{~d}$ after the burst (Frail \& Soderberg 2005).

In the following we focus on the observations by the X-Ray Telescope (XRT, Burrows et al. 2005a) on board Swift. In section 2 we describe the data analysis. In Section 3 we discuss on theoretical implications of these observations and in Section 4 we draw our conclusions.

\section{XRT AND UVOT OBSERVATIONS}

UVOT (Roming et al. 2005) observations started on Jan 28, 2005 at 20:03:07 UT, about 15.8 hours after the burst. This delay happened because UVOT was in safe mode at the trigger time. During each orbit UVOT acquired a single shot image in each filter $U, B$ and $V$ in imaging mode. UVOT did not detect

\footnotetext{
${ }^{1}$ INAF - Osservatorio Astronomico di Brera, Via Bianchi 46, I-23807 Merate (LC), Italy

2 INAF - Osservatorio Astronomico di Roma, Via di Frascati 33, I-00040 Monteporzio, Italy

${ }^{3}$ Università degli studi di Milano-Bicocca, Dipartimento di Fisica, Piazza delle Scienze 3, I-20126 Milano, Italy

${ }^{4}$ INAF - Istituto di Astrofisica Spaziale e Fisica Cosmica Sezione di Palermo, Via Ugo La Malfa 153, I-90146 Palermo, Italy

${ }^{5}$ International School for Advanced Studies (SISSA-ISAS), Via Beirut 2-4, I-34014 Trieste, Italy

${ }^{6}$ Department of Astronomy \& Astrophysics, 525 Davey Lab., Pennsylvania State University, University Park, PA 16802, USA

${ }^{7}$ ASI Science Data Center, Via Galileo Galilei, I-00044 Frascati (Roma), Italy

${ }^{8}$ NASA/Goddard Space Flight Center, Greenbelt, MD 20771

${ }^{9}$ Department of Physics and Astronomy, Johns Hopkins University, 3400 North Charles Street, Baltimore, MD 21218

${ }^{10}$ Center for Gravitational Wave Physics, Pennsylvania State University, University Park, PA 16802, USA

${ }^{11}$ Department of Astronomy, University of Texas, RLM 15.308, Austin, TX 78712-1083

12 Department of Physics, University of Nevada, Box 454002, Las Vegas, NV 891, USA

${ }^{13}$ Department of Physics and Astronomy, University of Leicester, Leicester LE 1 7RH, UK

${ }^{14}$ Mullard Space Science Laboratory, University College London, Holmbury St. Mary, Dorking, RH5 6NT Surrey, UK
} 
the afterglow. Upper limits on the first (summed over about 1 d elapsed time) UVOT observations are $V<18.9(V<19.8)$, $B<20.3(B<21.1)$ and $U<19.2(U<20.3)$.

XRT observed GRB050128 after an automatic slew of the Swift satellite. However, XRT was not yet operating in its nominal automatic mode changing configuration but rather in a manual mode for the purpose of obtaining calibration data. Before pointing to GRB050128, the XRT was observing a UVOT calibration target in photon counting mode (see Hill et al. 2004 for a description of XRT observing modes). XRT fully settled on the BAT position $108 \mathrm{~s}$ after the trigger. XRT observed GRB050128 for 17 orbits following the first pointing, accumulating a nominal exposure time of $17303 \mathrm{~s}$ (distributed over 73 ks). This low Earth orbit of Swift causes source observations to be interrupted each orbit. At the same time, thanks to the fastpointing capability of Swift, several targets may be observed per orbit. At this early stage of the mission the analysis of the data is not straightforward. We analysed the data running the task xrtpipeline within FTOOLS v5.3.1 and cutting out temporal intervals when the CCD temperature was higher than $-50^{\circ}$ Celsius (see Burrows et al. 2005b) and when the total count rate in the $0.2-10 \mathrm{keV}$ energy band over the entire CCD was larger than $85 \mathrm{c} \mathrm{s}^{-1}$ (these counts are mainly soft counts and are due either to dark current or to the bright Earth limb near the end of each snapshot observation). With these cuts we obtain a total exposure time of $13047 \mathrm{~s}$ distributed over 11 orbits.

\subsection{Angular analysis}

A fading source is clearly evident in all the XRT orbits. In the first two orbits the source is clearly piled-up and to derive an unbiased position we rely on the remaining $\sim 10 \mathrm{ks}$ exposure. An image has been extracted in the $0.5-10 \mathrm{keV}$ energy band to avoid contamination from low energy photons. The source position has been derived with XIMAGE (v4.2.1) using the centroid command: RA(J2000): $14 \mathrm{~h} 38 \mathrm{~m} 18 \mathrm{~s} .0$, $\operatorname{Dec}(\mathrm{J} 2000)$ : $-34^{\circ} 45^{\prime} 55^{\prime \prime}$.9. The main contributors to the positional uncertainty are the uncalibrated satellite attitude and boresight, resulting in $a \sim 6^{\prime \prime}$ error radius $(90 \%$ confidence level).

\subsection{Temporal analysis}

In order to properly track the decay of the fading source we have to account for the piled-up core in the first two snapshot observations. To this aim we extracted photons from an annular region (inner and outer radii 4 and 30 pixels, respectively) on source. This aperture was then applied to the rest of the observations, even when it was not needed. The light curve will have an underestimated normalization but it will not be distorted by pile-up. A background light curve has also been extracted from an annular region (inner and outer radii 80 and 120 pixels, respectively) centered on the same position and free of other sources and hot pixels. Fig. 1 shows the background-subtracted light curve in the $0.2-10 \mathrm{keV}$ energy band. The source is clearly fading. The decay light curve is not consistent with a single power law $\left(\chi_{\text {red }}^{2}=2.6\right.$, with 35 degrees of freedom, dof, null hypothesis probability, nhp, $7 \times 10^{-7}$ ), being flat at the beginning and steepening at later times. We also tried a single power law with a different initial time $t_{0}$, considered here as a free parameter. We can account in principle for the observed decay with $t_{0}=-780 \pm 290 \mathrm{~s}$, however this early time is not physically meaningful. A better description of the data is provided by two power laws (with indices $\alpha_{1}$ and $\alpha_{2}$ ) smoothly joined at a break time $t_{b}$ to model the decay. The fit with this model is $\operatorname{good}\left(\chi_{\text {red }}^{2}=0.7\right.$ with 33 dof, $\left.0.90 \mathrm{nhp}\right)$ and $\alpha_{1}=-0.27_{-0.12}^{+0.10}$, $\alpha_{2}=-1.30_{-0.13}^{+0.18}$ and $t_{b}=1472_{-290}^{+300} \mathrm{~s}$ (these errors are $90 \%$ for one interesting parameter, i.e. $\Delta \chi^{2}=2.71$, throughout the letter).

\subsection{Spectral analysis}

Given the large variability in the source count rate and the knowledge from the temporal analysis of the existence of a change in the decay slope, we extracted three spectra from our data, one from each of the first two snapshots and one for the rest of the observation (see Fig. 1). The first two spectra were extracted from the same annular region as for the temporal analysis. The last spectrum, since the source is much fainter, was extracted from a circular region on source of 30 pixel radius. Exposure times are 286, 1653 and 10731 s, respectively. Data have been filtered for grades 0-4 (according to the XMMNewton nomenclature, i.e. single and double pixel events ${ }^{15}$ ), since at this stage the response matrix for the standard 0-12 pixel event is not fully calibrated. Ancillary response files were generated with the task xrtmkarf within FTOOLS (v5.3.1), accounting for the different extraction regions. Data were rebinned to have at least 20 counts per energy bin and allow $\chi^{2}$ fitting within XSPEC (v11.3.1).

We fit the data with an absorbed power law model for all the observations. We first fixed the absorbing column density to the Galactic value of $N_{H}=4.8 \times 10^{20} \mathrm{~cm}^{-2}$. We added a $5 \%$ systematic uncertainty to all our fits to account for the residuals still present in our response matrix (given the relatively low number of counts this does not alter our results sensibly). This simple fit can account for the observed spectra. The fit is good with $\chi_{\text {red }}^{2}=1.1$ for 63 dof $(0.21 \mathrm{nhp})$. The power law photon index is $\Gamma=1.66_{-0.07}^{+0.06}$. The $0.2-10 \mathrm{keV}$ unabsorbed fluxes of the three observations with mid times of $258 \mathrm{~s}, 6156 \mathrm{~s}$ and $51587 \mathrm{~s}$ are $2.2 \times 10^{-10}, 1.9 \times 10^{-11}$ and $6.6 \times 10^{-13}$ erg $\mathrm{s}^{-1} \mathrm{~cm}^{-2}$, respectively. Given the slope change in the light curve we also untie the power law photon index of the three observations (even if it is not required by the statistics). The three photon indices are $1.59 \pm 0.08,1.79 \pm 0.11$ and $1.59 \pm 0.23$, respectively. These values are consistent with each other with a small deviation in the second snapshot. Although the fit is consistent with the Galactic column density, we let the column density value free to vary. The fit is improved with a $\chi_{\text {red }}^{2}=0.9$ (62 dof, $0.66 \mathrm{nhp}$, see Fig. 2) and the improvement is significant according to the F-test (probability $4 \times 10^{-4}$, even if we improved an already statistically good fit). In Fig. 3, we show the contour plot of the column density vs. power law photon index. The fit obtained with the column density fixed to the Galactic value is outside the $3 \sigma$ boundary. The absorbing column density is $(1.0 \pm 0.2) \times 10^{21} \mathrm{~cm}^{-2}$ and the power law photon index $\Gamma=1.88 \pm 0.12$. Unabsorbed fluxes $(0.2-10 \mathrm{keV})$ are $2.4 \times 10^{-10}, 2.0 \times 10^{-11}$ and $7.0 \times 10^{-13} \mathrm{erg} \mathrm{s}^{-1} \mathrm{~cm}^{-2}$, respectively. Also in this case, leaving free the photon index to vary within the observations the second one is characterized by a slightly steeper index.

\section{PHYSICAL INTERPRETATIONS}

15 See e.g. the XMM-Newton User's Handbook at http://xmm.vilspa.esa.es/external/xmm_user_support/documentation/uhb/index.html 
The major result on the GRB050128 afterglow concerns the monitoring in the X-ray band of its early temporal decay. This decay cannot be described by a simple power law but can be accounted for by a slowly varying double power law decay. During this transition there are no apparent marked spectral changes. The most straightforward interpretation is that the temporal break reveals a jet, i.e. corresponding to the epoch when the relativistic beaming angle $(1 / \gamma)$ becomes larger than the physical opening angle $\left(\theta_{j}\right)$ of the jet during the fireball deceleration (e.g. Rhoads 1999). In the slow cooling regime, for a uniform density circumstellar medium, the temporal decay changes from $t^{3(1-p) / 4}$ to $\sim t^{-p}$ (e.g. Rhoads 1999), which is well consistent with the observed temporal decay indices when $p \sim 1.3$ is adopted. In such a case no spectral change is expected. However, the expected spectral photon index should be $-(p+1) / 2 \sim-1.15$, too small to be compared with the observed value. In order to make the jet model work, one needs to assume $\nu_{c}<\nu_{X}<\nu_{m}$ before the jet break, and $\nu_{X}>\max \left(\nu_{c}, \nu_{m}\right)$ after the jet break (here $\nu_{m}$ and $\nu_{c}$ are the typical synchrotron frequency and the cooling frequency, respectively). In such a case, $p \sim 1.3$ gives a consistent interpretation of both spectral and temporal indices in all three epochs, regardless of whether the medium is an interstellar medium (ISM) or a wind from a massive companion. This model requires a little bit of coincidence in that the synchrotron frequency happens to cross the X-ray band during the jet break. However, considering the rapid decline with time of $\nu_{m}$ this is not a very unlikely possibility. Another caveat is that a flat electron spectrum $p \sim 1.3$ is abnormal in late afterglow fits (e.g. Panaitescu \& Kumar 2001). However, since we are observing a previously unexplored early epoch, a small $p$ required for the jet model to work cannot be ruled out. Possible ways to generate a flat electron spectrum have been suggested earlier (e.g. Bykov \& Mészáros 1996). If this is indeed a jet break, this would be the earliest jet break detection so far. Using the standard definition of jet break time (i.e. $\theta_{j}=1 / \gamma\left(t_{j}\right)$ ) one can derive $\theta_{0}=1.8^{\circ}\left(t_{j} / 2000 \mathrm{~s}\right)^{3 / 8}\left(E_{52} / n\right)^{-1 / 8}[(1+$ $z) / 2]^{-3 / 8}$ for a constant density interstellar medium and $\theta_{0}=$ $3.7^{\circ}\left(t_{j} / 2000 \mathrm{~s}\right)^{1 / 4}\left(E_{52} / A_{*}\right)^{-1 / 4}[(1+z) / 2]^{-1 / 4}$ for a wind model. Here $n$ is the density of the ISM, $E_{52}$ is the isotropicequivalent burst energy in units of $10^{52} \mathrm{erg}$ and $A=\dot{M} /(4 \pi v)$ is the wind parameter, with $\dot{M}$ being the mass loss rate, $v$ being the wind velocity, and $A_{*}=A /\left(5 \times 10^{11} \mathrm{~g} \mathrm{~cm}^{-1}\right)$. These jets are not extremely narrow (e.g. Covino et al. 2003) but are narrower than the typical jets identified in the previous late afterglow observations (e.g. Table 2 of Bloom et al. 2003). According to the GRB standard energy argument (e.g. Frail et al. 2001; Panaitescu \& Kumar 2001), such a narrow jet should correspond to large isotropic gamma-ray energy. Since this burst was not particularly bright, it might lie in the low energy tail of GRB-energy distribution, thus being another outlier for the standard energy relation.

Besides the jet interpretation, one could search for other possible solutions by considering the temporal and spectral relations in various afterglow models (e.g. Mészáros, Rees \& Wijers 1998; Sari, Piran \& Narayan 1998; Chevalier \& Li 2000; Zhang \& Mészáros 2004). The most straightforward model is within the framework of the standard isotropic wind model (Chevalier \& Li 2000). The first cluster of the data corresponds to the $\nu_{c}<\nu_{X}<\nu_{m}$ regime, in which the temporal index $-1 / 4$ and the photon spectral index $-3 / 2$ are expected. The second and the third clusters of the data correspond to the regime of $\nu_{m}<\nu_{X}<\nu_{c}$, in which the temporal index $-(3 p-1) / 4$ and the photon spectral index $-1-(p-1) / 2$ are expected. All these are consistent with the data for $p \sim 2.1$. In this interpretation, one needs to assume that both $\nu_{m}$ and $\nu_{c}$ cross the X-ray band during the gap between the first two clusters of data and that the frequencies switch the order. This could be achieved with a small wind parameter (e.g. $A_{*}$ in the range of $0.01-0.001)$. One caveat is that in the wind model $\nu_{c} \propto t^{1 / 2}$, so that the time interval of the gap is not long enough for $\nu_{c}$ to completely cross the band. Nonetheless, the spectral slope in the second cluster is slightly steeper than the other two, which might be still consistent with the data if one introduces an evolving cooling break near the high energy edge of the band during the epoch of the second snapshot. Introducing a slightly steeper density profile (larger than $r^{-2}$ for the wind case) could further alleviate the problem. Furthermore, if the electron equipartition factor $\epsilon_{B}$ drops during the temporal gap, this would speed up the $\nu_{c}$ crossing time scale, making the model more consistent with the data.

Finally, we note also that a similar behaviour has been observed in optical light curve GRB021004 even if with a slightly longer break time $(\sim 0.1 \mathrm{~d}$, Fox et al. 2003). This transition has been interpreted by Li \& Chevalier (2003) as a fast to slow cooling transition.

\section{CONCLUSION}

Swift is exploring for the first time the early stages of GRB afterglow decays. We detect a clear early temporal break in the X-ray afterglow of GRB050128, with the spectral indices not changing around the break. The data could be argued to be consistent with either a jet model or a wind model. The jet model requires a flat electron spectrum and an assumed spectral domain change within the temporal gap between the first two snap shot observations. If this is true, we may have detected the earliest jet break so far. The wind model requires a (relatively) low ambient density and possibly an evolution of the $\epsilon_{B}$ parameter. We note that in this last case, passages from fast to slow cooling regimes might have remained hidden in the great majority of GRB afterglows if they are characterized by such a tenuous environment, due to the late times at which they were observed.

The early detection of the X-ray afterglow, coupled with the initial flat decay, allows us to estimate its fluence $\mathcal{F}=$ $\int F(t) \mathrm{d} t$. In fact, given the value of $\alpha_{1}$ and $\alpha_{2}, \mathcal{F}$ is not very sensitive to the start time $t_{0}$ of the afterglow and is dominated by the flux at the break time $t_{b}$. The fluence between $108 \mathrm{~s}$ and $73000 \mathrm{~s}$ is $(7 \pm 2) \times 10^{-7} \mathrm{erg} \mathrm{cm}^{-2}(15-350 \mathrm{keV})$, while extrapolating from $t=0$ to $+\infty$ it is $\mathcal{F}=9_{-3}^{+5} \times 10^{-7} \mathrm{erg} \mathrm{cm}^{-2}$. These values amount to $15 \%$ and $20 \%$ of the prompt fluence in the same energy band. Since prompt and afterglow spectra are similar we might expect a relatively small difference in the bolometric correction. This is the first determination of the ratio between GRB proper and early afterglow energetics.

This work is supported at OAB by funding from ASI on grant number I/R/039/04, at Penn State by NASA contract NAS500136 and at the University of Leicester by PPARC on grant numbers PPA/G/S/00524 and PPA/Z/S/2003/00507. We gratefully acknowledge the contributions of dozens of members of the XRT and UVOT team at OAB, PSU, UL, GSFC, ASDC, MSSL and our subcontractors, who helped make this instrument possible. 
REFERENCES

Antonelli, L.A., et al. 2005, GCN 2991

Barthelmy, S.D., et al. 2005, Sp.Sc.Rev. in press

Bloom, J.S., Frail, D.A., Kulkarni, S.R. 2003, ApJ, 594, 674

Burrows, D.N., et al. 2005a, Sp.Sc.Rev. in press

Burrows, D.N., et al. 2005b, ApJ in press (astro-ph/0502326)

Bykov, A.M., Mészáros, P. 1996, ApJ, 461, L37

Chevalier, R.A., Li, Z.Y. 2000, ApJ, 536, 195

Covino, S., et al. 2003, A\&A, 404, L5

Covino, S., et al. 2005, GCN 2999

Cummings, J., et al. 2005, GCN 2992

Fox, D.W., et al. 2003, Nat, 422, 284

Frail, D.A., et al. 2001, ApJ, 562, L55
Frail, D.A., Soderberg, A.M. 2005, GCN 3011

Gehrels, N., et al. 2004, ApJ, 611, 1005

Hill, J.E., et al. 2004, SPIE, 5165, 217

Li, Z., Chevalier, R.A. 2003, ApJ, 589, L69

Melandri, A., et al. 2005, GCN 3004

Mészáros, P., Rees, M.J., Wijers, R.A.M.J. 1998, ApJ, 499, 301

Monfardini, A., et al. 2005, GCN 3001

Panaitescu, A., Kumar, P. 2001, ApJ, 560, L49

Rhoads, J.E. 1999, ApJ, 525, 737

Roming, P.W.A., et al. 2005, Sp.Sc.Rev. in press

Sari, R., Piran, T., Narayan, R. 1998, ApJ, 497, L17

Zhang, B., Mészáros, P. 2004, Int.J.Mod.Phys., 19, 2385 


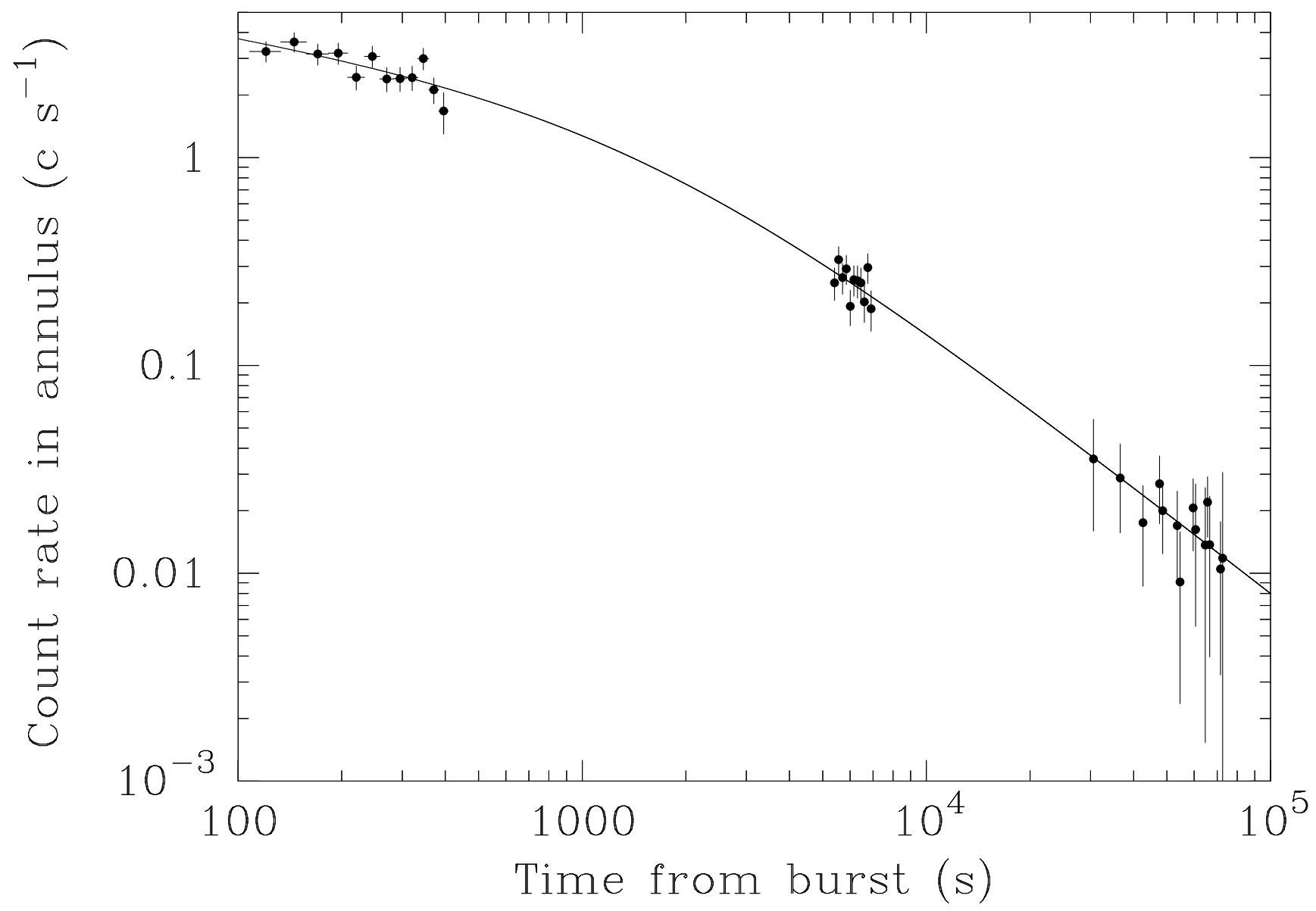

FIG. 1.- XRT 0.2-10 keV light curve extracted from an annular region centered on GRB050128. The continuous line represents the fit with two power laws smoothly joined. The conversion factor to translate the count rate into a $0.2-10 \mathrm{keV}$ unabsorbed flux is $8.7 \times 10^{-11} \mathrm{erg} \mathrm{cm}^{-2} \mathrm{counts}^{-1}$ (for a freely varying absorbed power law model, see text). 


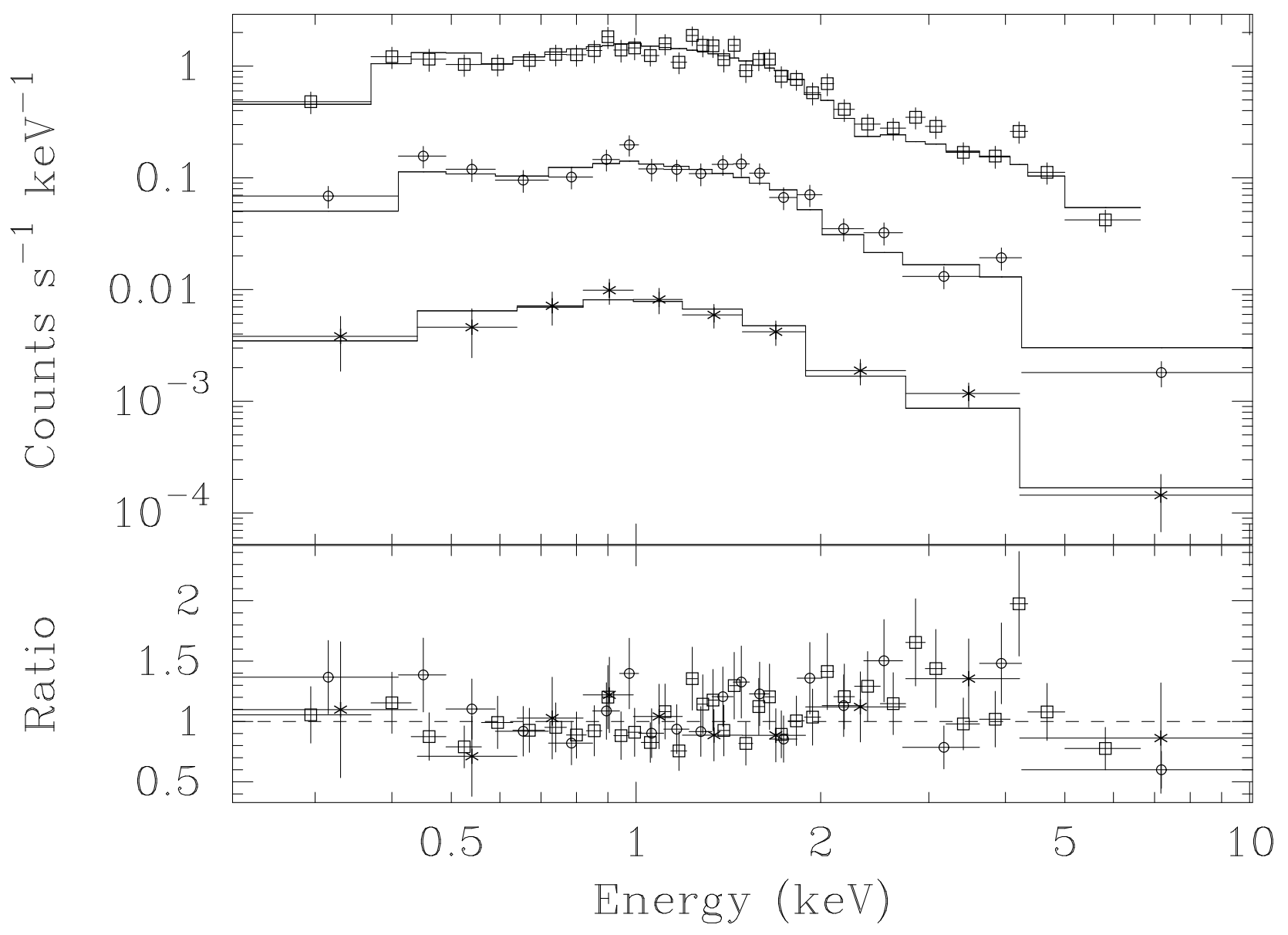

FIG. 2.- XRT 0.2-10 keV energy spectrum of GRB050128. In the upper panel are plotted the spectra of the three snapshot observations described in the text (1: squares, 2: circles, other: stars) fit with an (freely) absorbed power law model. In the lower panel there are the residuals from the same power law fit to all the data. 


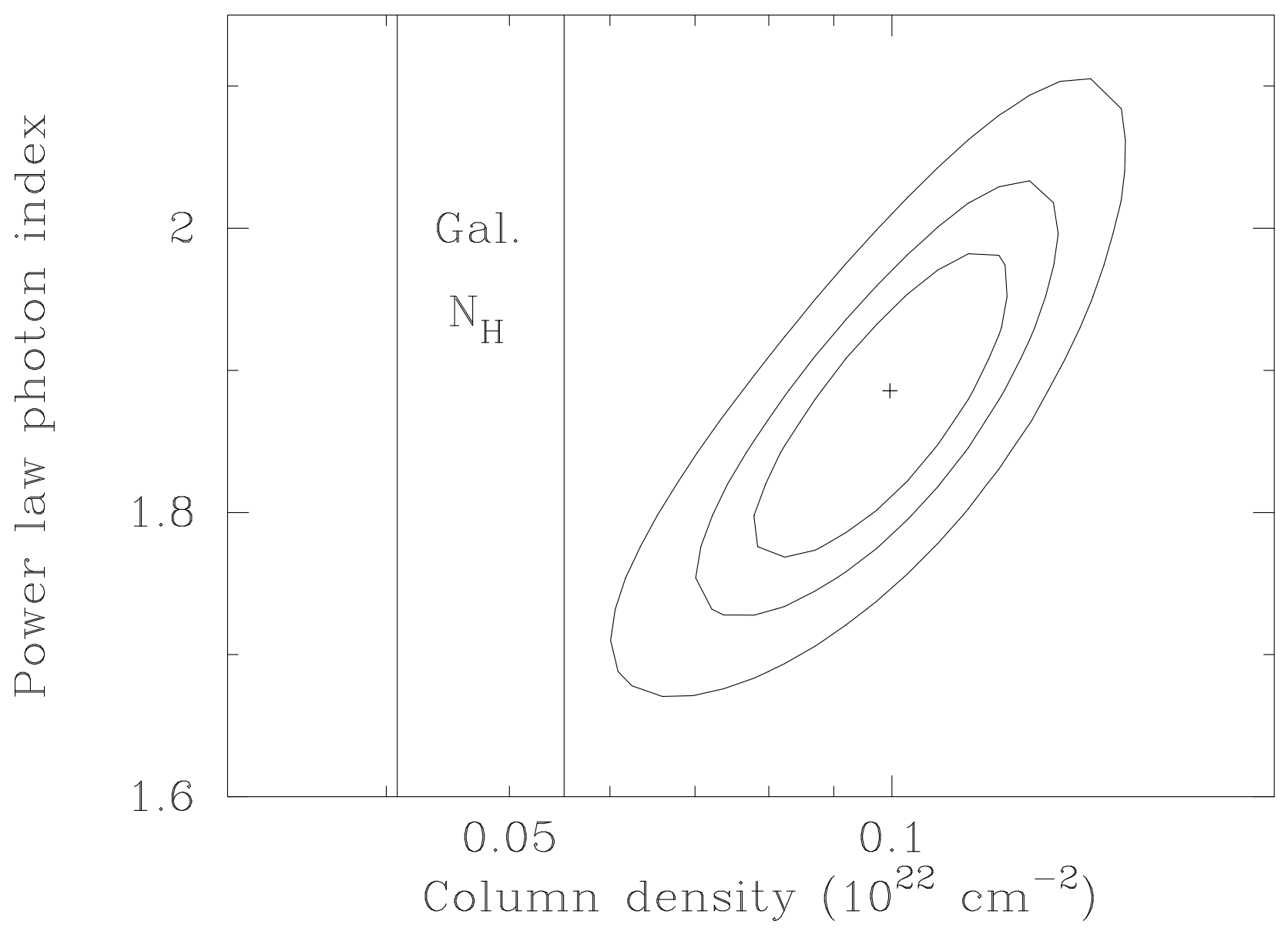

FIG. 3.- Contour plot of the column density versus the power law photon index for the X-ray spectrum of GRB050128. Contours refer to 1,2 and $3 \sigma$ confidence level. At the left of the contour plot the Galactic column density interval is drawn centered on the value of $4.8 \times 10^{20} \mathrm{~cm}^{-2}$ and with a $15 \%$ uncertainty. 